\title{
Center vortices of Yang-Mills theory at finite temperatures*
}

\author{
K. Langfeld, O. Tennert, M. Engelhardt and H. Reinhardt \\ Institut für Theoretische Physik, Universität Tübingen \\ D-72076 Tübingen, Germany
}

\begin{abstract}
Recent lattice calculations performed at zero temperature and in the maximal center gauge indicate that quark confinement can be understood in this gauge as due to fluctuations in the number of magnetic vortices piercing a given Wilson loop. This development has led to a revival of the vortex condensation theory of confinement. For a $\mathrm{SU}(2)$ gauge group, we show that also at finite temperatures, center vortices are the relevant collective infrared degrees of freedom determining the long-range static quark potential; in particular, their dynamics reflect the transition to the deconfining phase.
\end{abstract}

* Supported in part by DFG under contract Re 856/1-3. 
Introduction. One of the most intriguing prospects of strong interaction physics is the expected existence of a deconfined phase above a certain transition temperature. While strongly interacting matter, i.e. matter carrying a color quantum number, has to date only been observed in the form of color-singlet bound states called hadrons, the deconfined phase is characterized by the possibility of colored constituents propagating freely over distances large compared with hadronic sizes. Upcoming experiments at RHIC and LHC are expected to probe the deconfined regime, and thus efforts to establish an adequately detailed theoretical picture of the deconfinement phase transition and the deconfined phase acquire a measure of urgency.

The investigation of the deconfinement transition is irrevocably tied to the question of the mechanism leading to confinement in the low-temperature phase of QCD, the gauge theory of the strong interactions. It should be noted that in different gauges, different characteristic infrared collective degrees of freedom dominate the physics of confinement, and the convenience of a gauge hinges on the ease with which these infrared degrees of freedom can be identified and isolated. Examples of gauges which are in this sense convenient are the maximal Abelian gauge, which displays monopole dominance and thus underpins the dual superconductor picture of confinement [1]-[1], and the maximal center gauges [5]-[9], which display center dominance and lead to the center vortex picture of confinement [5]-[16]. It is this latter picture we concentrate on in this letter.

Maximal center gauges are defined in the lattice formulation of Yang-Mills theory by the requirement to choose link variables on the lattice as close to center elements of the gauge group as the gauge freedom will allow. There are many slightly differing ways of implementing this general idea; in this work, the specific prescription called "direct maximal center gauge" was adopted, for details see e.g. [7]. Furthermore, in what follows, we will be concerned with an $\mathrm{SU}(2)$ gauge group, the center of which consists of the elements \pm 1 . The maximal center gauges have the following convenient property: If one replaces the gauge-fixed link variables with the center elements closest to them (so-called center projection), then a Monte Carlo calculation of the long-range zero-temperature static quark potential via the Wilson loop yields virtually the same result as when using the full unprojected link variables [5]. This empirical observation is called center dominance. Note that in both cases, the weight in the Monte Carlo calculation is the full Yang-Mills action; only the observable, i.e. the Wilson loop, is sampled using either the full or the projected configurations. Center dominance is interpreted as meaning that the maximal center gauges successfully concentrate the information relevant for confinement on the center part of the link variables, thereby rendering the projection procedure of discarding the residual deviations from the center elements inconsequential. 
In order to further physically interpret the center projected link configurations, one can connect them with a notion of vortices as follows. After center projection, one is left with lattice links associated with center elements \pm 1 of the gauge group $\mathrm{SU}(2)$. If the links bordering any given plaquette on the lattice multiply to -1 , then a vortex is said to pierce that plaquette. The vortices defined in this way form closed two-dimensional surfaces in a four-dimensional space-time lattice, or closed (magnetic flux) lines on the three-dimensional lattice describing, say, one time slice. An important property of these vortices is that they contribute a factor -1 to any Wilson loop whenever they pierce its minimal area; in this way, they produce the same value for the Wilson loop as one obtains by multiplying the original centerprojected links making up the loop (this is simply Stokes' theorem). If a Wilson loop area is pierced by vortices an even number of times, then it takes the value +1 ; if a Wilson loop area is pierced by vortices an odd number of times, then it takes the value -1 . Thus, if the distribution of vortices in space-time fluctuates sufficiently randomly, then a strong cancellation will occur between configurations where Wilson loops are linked by an even or by an odd number of vortices; an ever better cancellation as the area of the Wilson loop increases leads to an area law decay of the Wilson loop expectation value, which implies a linear static quark potential. This is the essence of the random vortex picture of confinement. After having been proposed as early as 1978 [11]-[13], glimpses of such vortex configurations were afforded by the Copenhagen "spaghetti" vacuum [14], and several investigations were devoted to identifying vortices on the lattice, leading to different definitions of vortices [15, [16], [5]. The recent efforts in the framework of the maximal center gauges outlined above [5]- [9] have given new impetus to this particular description of confinement, foremost due to the verification of center dominance [5]. Subsequently, further properties of center vortices have been investigated, e.g. the connection to the monopoles of the maximal Abelian gauge [6], and the observation that the density and binary correlations of these vortices display the correct renormalization group scaling properties [8], [9]. This means that they survive the continuum limit and represent physical objects rather than lattice artifacts.

Having identified such a viable picture of confinement, it is natural to confront it with the question of the deconfinement transition. In this letter, we verify that the center vortex picture makes sense also at finite temperature, since center dominance persists. Also the transition to the deconfined phase with a vanishing string tension is correctly reproduced. We further explore properties of vortices as the temperature is raised across the deconfinement transition and we cast the deconfinement order parameter into the vortex language.

Zero temperature vortex properties. For later reference and in order to enable the reader to estimate the accuracy which is achieved in our numerical calculations, we briefly summarize our zero temperature results. Our numerical data were 


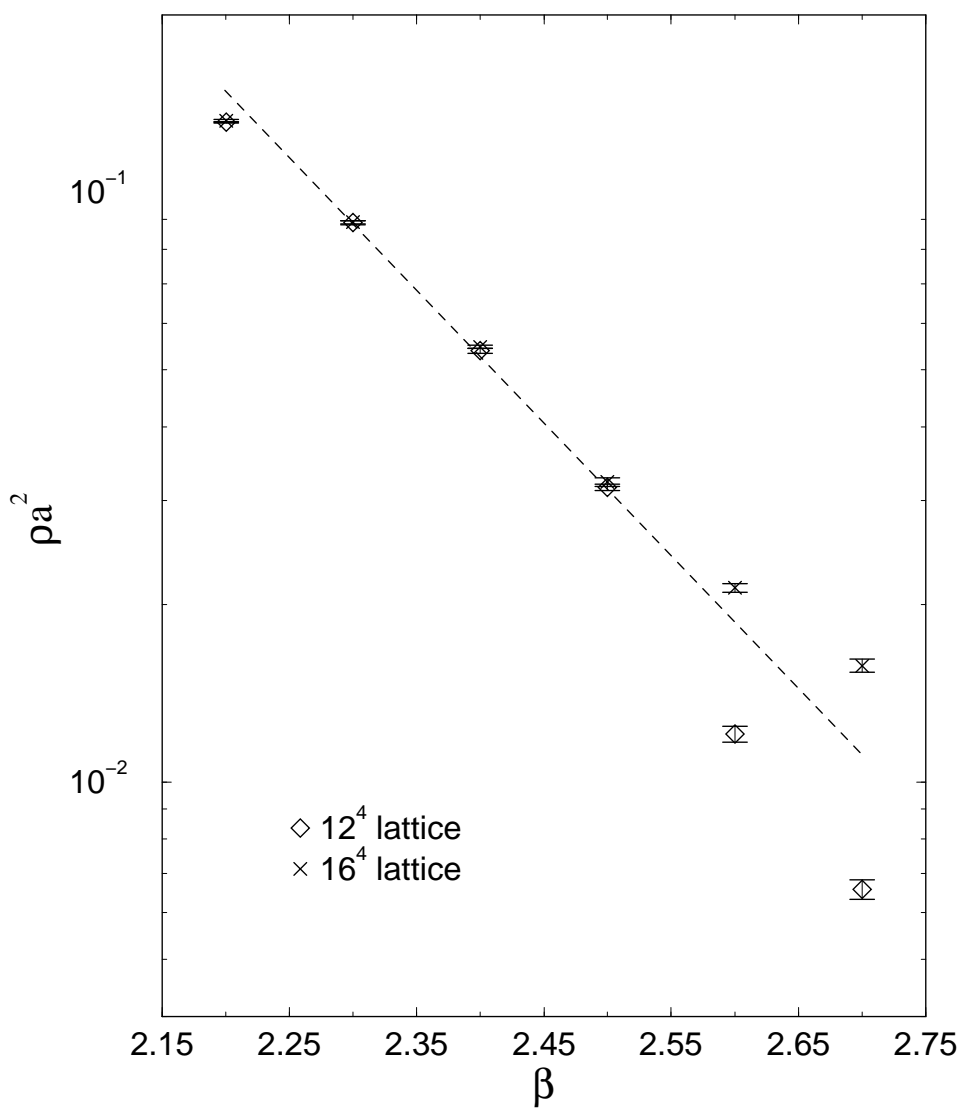

Figure 1: Scaling of the planar density of vortex intersection points with a given space-time plane.

obtained using lattices of size $12^{4}$ and $16^{4}$. The string tension is extracted from center-projected links by calculating $n \times n$ Creutz ratios, $n=3,4,5$. One observes that the results for the Creutz ratios for different $n$ agree already for these small $n$, indicating that the Coulomb part of the heavy quark potential is absent when the latter is evaluated using center-projected links [5]. The Creutz ratios allow us to extract $\sigma a^{2}(\beta=2.3)=0.12(3)$. The $\beta$-dependence of $\sigma a^{2}$ is compatible with oneloop perturbative scaling. Two-loop effects are small compared with the statistical error of our numerical data. Furthermore, for the lattices used $\left(12^{4}\right.$ and $\left.16^{4}\right)$, the finite size dependence of dimensionless quantities was negligible compared with the statistical errors. However, as is well known, considerable finite size effects persist in the extraction of the mass scale, as e.g. encoded in the lattice spacing, as a function of $\beta$. In the large scale analysis [10], an interpolation of the numerical data with the 
help of one-loop scaling to the renormalization point $\beta=2.3$ reveals $\sigma a^{2}=0.136$ for a $10^{4}$ lattice, $\sigma a^{2}=0.121$ for a $16^{4}$ lattice, while $\sigma a^{2}=0.107$ is found for a $32^{4}$ lattice. Compatible with this, we use throughout this letter $\sigma a^{2}(\beta=2.3)=0.12$ and $\sigma=(440 \mathrm{MeV})^{2}$ as reference scale for assigning physical units to measured quantities. When finite size uncertainties are mentioned below, this always refers to the aforementioned difficulty in defining the mass scale at a given $\beta$.

In order to extract vortex properties, we fix the configurations to the direct maximal center gauge by using the procedure described in [7]. To eliminate Gribov copies, we perform random gauge transformations and repeat minimizing the gauge fixing functional. It turns out that three of these runs are sufficient at zero temperature [7]. For temperatures close to the transition point, we observe an increasing influence of the Gribov copies making six gauge fixing iterations necessary. The scale dependence of the vortex density is shown in figure 1. We find a perfect scaling of the planar density $\rho$ of vortex intersection points with a given space-time plane in the range $\beta \in[2.25,2.55]$ for lattice sizes $12^{4}, 16^{4}$. We finally extract $\rho=3.6 \pm 0.2 \mathrm{fm}^{-2}$. The large error bar in $\rho$ results from the uncertainty in the magnitude of finite size effects. The above value, which is larger than the one quoted in [8], is now in agreement with the one quoted in [7] within the error bars. The faulty estimate for $\rho$ given in [8] is due to the fact that the Coulomb part of the full zero temperature static quark potential was underestimated in [8], which subsequently led to an overestimate of the reference scale. As a consequence, mass scales in [8], and also in [9], should be rescaled upwards by a factor 1.35. Note also that the new corrected value of $\sigma / \rho=1.4 \pm 0.1$ as opposed to the old value $\sigma / \rho=2.5$ implies that a model of random intersection points of vortices with a two-dimensional space-time plane [9] overestimates, rather than underestimates, the string tension as a function of the planar density of points $\rho$. Such a model [9] leads to a value of $\sigma / \rho=2$. However, this does not affect the motivation for the lattice measurements in 99 nor their validity, up to the aforementioned trivial rescaling of mass scales. In 9], correlations of an attractive type were observed between intersection points of vortices with a two-dimensional space-time plane. It seems plausible that such correlations curtail the randomness of the distribution of the points and thus reduce the string tension. Indeed, further below it will become clear that it is a pairing of the vortex intersection points which ultimately leads to deconfinement. However, one should bear in mind that the connection between correlators such as measured in [9] and the string tension is very indirect and one can not in general predict the behavior of the string tension from these correlators alone.

Center dominance at finite temperatures. Finite temperature Yang-Mills theory can be cast into a path integral formulation on a space-time manifold of finite (Euclidean) time extension $1 / T$, where $T$ is the temperature. Gauge fields on the lattice obey periodic boundary conditions in the time direction. The static quark 
potential $V(r)$ is calculated from the correlator of two Polyakov loops [17],

$$
\begin{aligned}
\langle L(\vec{x}) L(\vec{y})\rangle & \propto \exp \{-V(r) / T\} \\
L(\vec{x}) & =\mathcal{P} \exp \left(i \int_{0}^{1 / T} A_{0}(t, \vec{x}) d t\right),
\end{aligned}
$$

where $A_{\mu}(x)$ is the gauge potential, $\mathcal{P}$ denotes path ordering, and $r=|\vec{x}-\vec{y}|$.

Note that, since center elements of the gauge group commute, the Polyakov loop correlator evaluated with center-projected configurations is equal to the Wilson loop of identical spatial width and extending along the entire time direction. Therefore, center vortices contribute to the static quark potential at finite temperatures in the same way as they do at zero temperature (i.e. Stokes' theorem applies in the same way). The question to be settled is whether, at finite temperature, vortices still provide the entire long-range static quark potential (center dominance) or whether other effects become important. This would mean that center vortices lose their role as relevant infrared collective degrees of freedom.

We have tested this empirically employing Monte Carlo measurements. On the one hand, we have evaluated Polyakov loop correlators using center-projected links on a $12^{3} \times N_{t}$ lattice with $N_{t}=5,6,7$ for different $\beta \in[2.26 \ldots 2.4]$, i.e. different inverse temperatures $1 / T=N_{t} a(\beta)$ were achieved by varying $\beta$. The $\beta$-dependence of the lattice spacing $a$ was extracted from zero temperature string tension measurements (see previous section). While these measurements are thus fraught with sizeable uncertainties due to finite size effects in $a(\beta)$, the statistical fluctuations still turn out to be the dominant source of error.

The center-projected string tension $\sigma_{t}$ as a function of temperature was extracted from the Polyakov loop correlators by fitting a linear law to the potential $V(r)$ of eq. (11). A Coulomb term is not necessary, since, as will be illustrated by an example below, center projection removes the perturbative Coulomb part from $V(r)$ just as at zero temperature [5]. In addition, however, it should be kept in mind that the static quark potential at finite temperatures in general also contains a logarithmic dependence on the separation [18]. Thus, fitting a purely linear law to the potential strictly speaking does not yield the coefficient of the linear term, which by definition constitutes the string tension; instead, one obtains an effective "string tension" which provides a good parametrization of the long-range static quark potential in the limited range of separations accessible to lattice experiments. Since the accuracy of our measurements is limited, we cannot separate the linear and logarithmic parts of the potential, and we thus quote instead the effective string tension in the sense explained above. This quantity in full Yang-Mills theory is known to behave as follows. It retains its zero-temperature value to within approximately $10 \%$ up to the temperature $0.8 T_{c}$, where $T_{c}$ is the deconfinement phase transition temperature, 

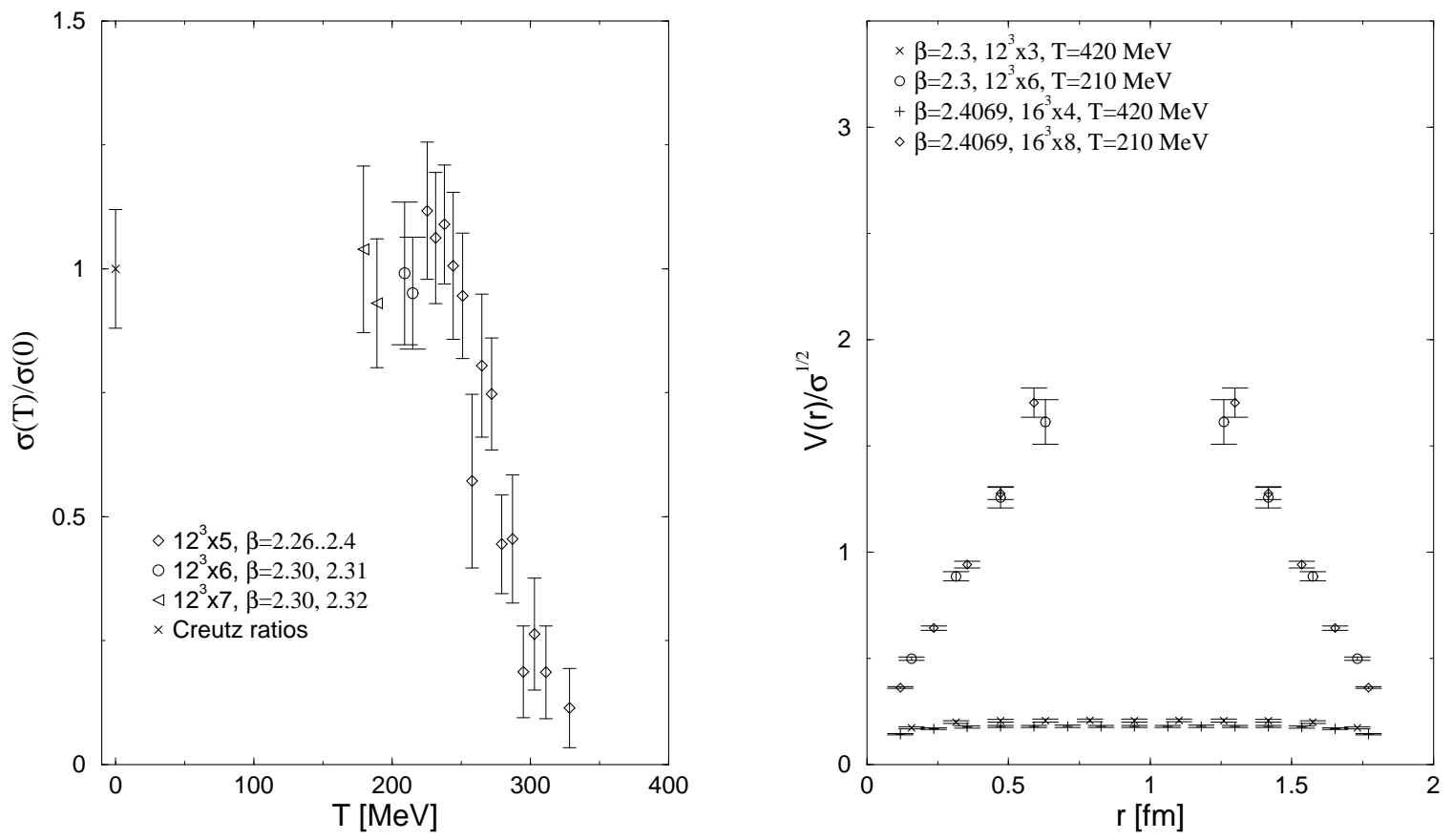

Figure 2: Left: The string tension $\sigma_{t}$ calculated from center-projected links as function of the temperature $T$; the four lowest-temperature points on the $12^{3} \times 5$ lattice are measured at $\beta<2.3$ and thus may already be subject to systematic scaling violations. The zero temperature measurement is included to give an indication of the error bars. Right: The static quark potential at two sample temperatures, as extracted from Polyakov loop correlators with center-projected links, in units of the zero-temperature string tension.

and then quickly drops to zero. For the SU(2) theory, we have only been able to find some rather sparse older data to substantiate this [19]; on the other hand, for the SU(3) case, which should behave qualitatively in the same manner, new high precision measurements exist [20] which corroborate the aforementioned behavior of the effective string tension.

Our results for the center-projected case are displayed in the left-hand plot of Figure 2. We find that the center-projected string tension $\sigma_{t}$ within the error bars reproduces the behavior of the full string tension quoted above; thus, we observe center dominance of the long-range part of the static quark potential at finite temperatures $T<T_{c}$ within the accuracy of our measurement and the limited range of separations available. 
Moreover, the center-projected string tension $\sigma_{t}$ signals the deconfinement phase transition to occur at $T_{c}=295 \pm 10 \mathrm{MeV}$, where the input scale was a zero temperature string tension of $\sigma=(440 \mathrm{MeV})^{2}$. This value of $T_{c}$ is in good agreement with the high precision measurements of [21]. Thus, also the transition to the deconfined phase with a vanishing string tension is accurately reproduced by the center-projected theory.

For further illustration, we also calculated Polyakov loop correlators using in particular $\beta=2.3$ for a $12^{3} \times N_{t}$ lattice and $\beta=2.4069$ for a $16^{3} \times N_{t}$ lattice while varying the number of lattice points $N_{t}$ in time direction in order to obtain different inverse temperatures $1 / T=N_{t} a(\beta)$. The $\beta$ values and lattice sizes in both cases correspond to a physical size of the spatial cube of $L_{s}=N_{s} a(\beta) \approx 1.9 \mathrm{fm}$, while the lattice spacing changes by a factor $a(\beta=2.3) / a(\beta=2.4069)=4 / 3$. This particular choice of lattice sizes and $\beta$-values therefore allows to test the scaling behavior of the lattice observables with vastly diminished fluctuations due to finite size effects. Of course, the overall uncertainty in the mass scale remains. In the case of Polyakov loop correlators, this does not turn out to be crucial; the statistical fluctuations are the main source of error. However, when measuring vortex densities (see next section), this method substantially improves the observed scaling properties, since the statistical errors are not as dominant.

Using the Monte Carlo results for center-projected Polyakov loop correlators on these lattices, the static quark potential as a function of the distance at diverse temperatures both below and above the deconfinement transition was extracted. Two examples, corresponding to $N_{t}=3,6$ for $\beta=2.3$ and $N_{t}=4,8$ for $\beta=2.4069$, are given in the right-hand plot in Figure 2. For $T<T_{c}$, the potential rises linearly even at small distances; as observed previously for the case of zero temperature [5], center projection removes the short range Coulomb interaction.

Vortex polarisation. Given this success of the vortex picture, the question of the nature of the deconfinement transition in this picture poses itself. One of the simplest hypotheses would appear to be the following: While the center vortices are by construction one lattice spacing thick, they represent smooth configurations in the original gauge fields before the gauge fixing and center projection procedure is applied, with a physical thickness in the continuum limit [5], [9]. Possibly vortices running perpendicularly to the time direction are simply too thick to fit into the space-time manifold of time extension $1 / T$ for $T>T_{c}$. This would mean that the planar density of points at which vortices pierce the area spanned by the two Polyakov loops entering the loop correlator vanishes. Vanishing density of such points precludes fluctuations in the number of such points, making an area law decay of the Polyakov loop correlator impossible. In order to test this scenario, we have measured the planar density $\rho_{t}$ of points at which vortices pierce an area extending 

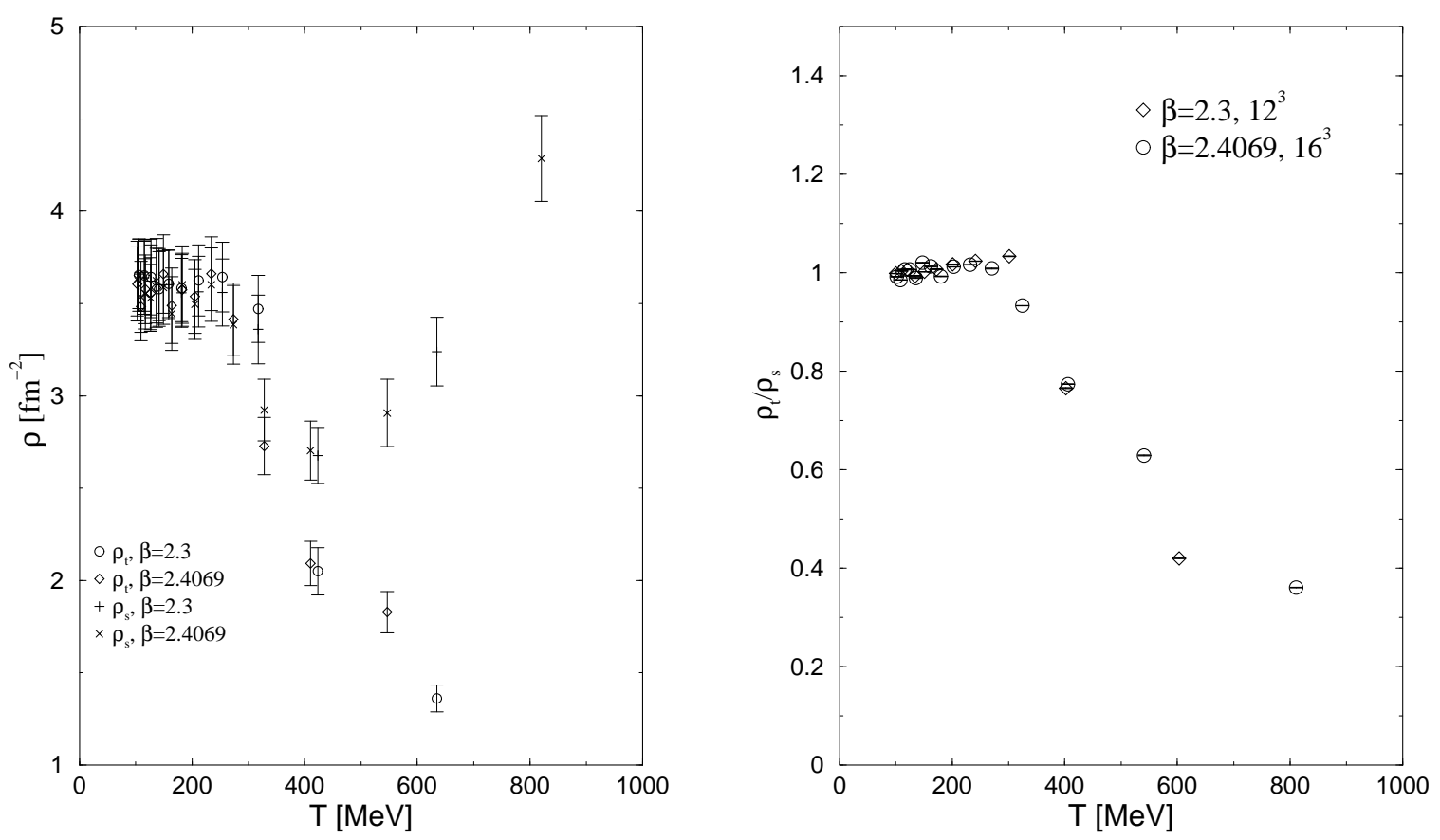

Figure 3: The planar densities $\rho_{t}$ and $\rho_{s}$ of points at which vortices pierce time-like and space-like planes, respectively.

in the time and one space direction, for different temperatures. In order to reduce fluctuations due to finite size effects (see previous section), these measurements were done using $\beta=2.3$ for a $12^{3} \times N_{t}$ lattice and $\beta=2.4069$ for a $16^{3} \times N_{t}$ lattice while varying the number of lattice points $N_{t}$ in time direction in order to obtain different inverse temperatures $1 / T=N_{t} a(\beta)$. The result for the vortex density is shown in Figure 3 .

Evidently, while $\rho_{t}$ experiences a drop as one increases the temperature past $T_{c}$, the behavior of $\rho_{t}$ is smooth and at $T \approx 2 T_{c}, \rho_{t}$ still retains roughly a third of its zero temperature value. Thus, the hypothesis advanced above of deconfinement being due to a vanishing of $\rho_{t}$ was too simplistic. There still exists a density of vortices piercing the area spanned by two Polyakov loops above $T_{c}$, but the random character of the distribution of these vortices must disappear in order to realize deconfinement. We will come back to this presently; before doing so, note that we have for comparison also measured the density $\rho_{s}$ of vortices piercing an area extending in two spatial directions. The ratio $\rho_{t} / \rho_{s}$ is also shown in figure 3. For low temperatures, $\rho_{t}$ and $\rho_{s}$ coincide, as they must do due to Euclidean $\mathrm{O}(4)$ invariance. At temperatures slightly above $T_{c}, \rho_{s}$ decreases along with $\rho_{t}$. At higher temperatures, however, $\rho_{s}$ 
begins to increase. This seems consistent with a simple picture of the intersection points making up the density $\rho_{s}$ still being distributed randomly, which leads to a linear relation [9] between the planar density $\rho_{s}$ and the corresponding spatial "string tension" $\sigma_{s}$ extracted from spatial Wilson loops. This spatial string tension in turn is known to increase as $\sqrt{\sigma_{s}} \sim g^{2}(T) T$ for $T \geq 2 T_{c}$ according to dimensional reduction arguments [22] and their verification in lattice experiments [21]. As mentioned above, in the case of the intersection points making up the density $\rho_{t}$, this simple random picture must by contrast become invalid above $T_{c}$.

Pairing of vortex intersection points. Coming back to the issue of the vortex description of the deconfinement transition, it is necessary to inspect more closely the properties of vortices piercing the area spanned by the two Polyakov loops entering a loop correlator. A necessary condition for an area law suppression of the loop correlator expectation value is an ever better mutual cancellation, as the area spanned by the Polyakov loops is increased, between configurations where the area is pierced an even or an odd number of times by vortices, respectively. We have therefore measured the probabilities of these two cases as a function of temperature for an area of spatial width $0.9 \mathrm{fm}$. For $\beta=2.3$, this corresponds to 6 lattice spacings in the $12^{3} \times N_{t}$ lattice, whereas for $\beta=2.4069$, it corresponds to 8 lattice spacings in the $16^{3} \times N_{t}$ lattice, i.e. in both cases the distance between the Polyakov loops is half the linear extension of the universe. The result of these Monte Carlo experiments is shown in Figure 4 , which displays the fraction $p(T)$ of cases where an area specified as above was pierced an even number of times by vortices. This quantity exhibits a sharp transition at the deconfinement temperature $T_{c} \approx 295 \mathrm{MeV}$. Note that $p \nrightarrow \rightarrow 1 / 2$ for large areas precludes an area law decay of the Polyakov loop correlator, implying deconfinement.

The clear signal of the deconfinement transition exhibited by the above probability $p$ is not surprising, since it constitutes nothing but a slightly more physical variant of the usual Polyakov loop order parameter. The center-projected Polyakov loop correlator is given as follows in terms of the probability $p$ that an even number of vortices pierces the area spanned by the two Polyakov loops in question:

$$
\langle L(\vec{x}) L(\vec{y})\rangle=p \cdot 1+(1-p) \cdot(-1)=2 p-1 .
$$

Thus, $p$ corresponds, up to a rescaling and a shift, to the Polyakov loop correlator itself. The only difference to the usual Polyakov loop order parameter, which is related to the free energy associated with placing a static color source in the thermal medium, is that the static color source has been given a partner (separated from it by half the extension of the universe).

Therefore, in defining the probability $p$, we have not really introduced a new, distinct, order parameter for the deconfinement transition. Rather, the vortex picture 


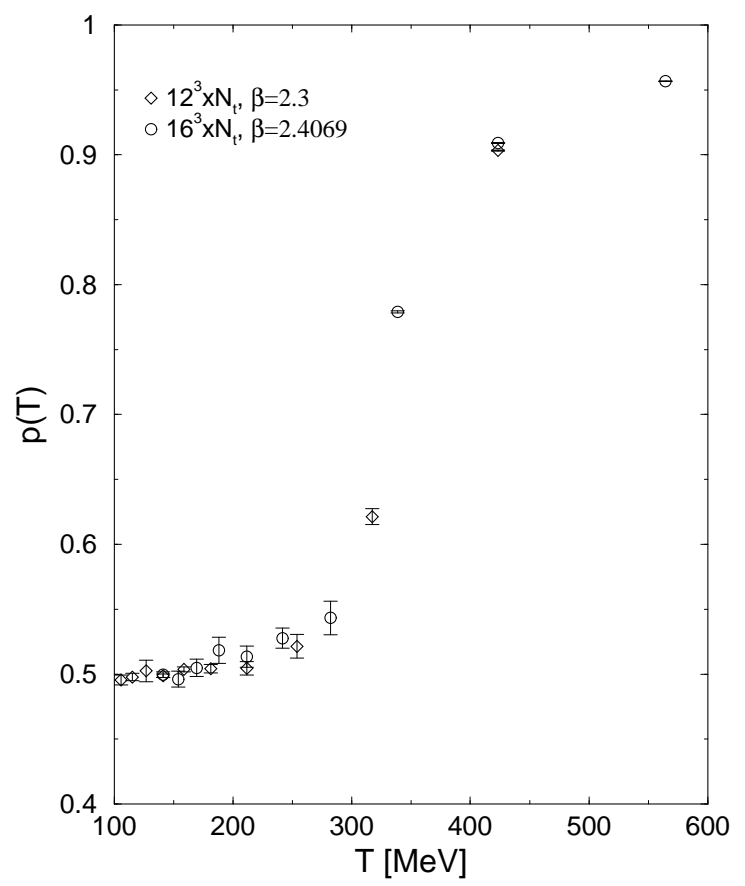

Figure 4: Fraction of cases in which an area spanned by two Polyakov loops was pierced an even number of times by vortices.

simply provides an alternative language for describing the Polyakov loop correlator, by characterizing the manner in which vortex intersection points occur on the area spanned by the two Polyakov loops. The deconfinement transition occurs when the intersection points of vortices with the area spanned by the Polyakov loops begin to occur predominantly in pairs of finite separation (above $T_{c}$ ), whereas they occur randomly below $T_{c}$.

Summary and Outlook. In the present work, we have explored the center vortex content of Yang-Mills theory at finite temperatures using numerical lattice methods in the direct maximal center gauge and center projection. We have observed vortex dominance for the long-renge part of the static quark potential. This establishes the relevance of center vortices at finite temperatures and thus provides the necessary basis for further investigation of these degrees of freedom.

The planar densities of intersection points of vortices with a given plane in spacetime exhibit an anisotropic behavior as temperature is raised from the $O(4)$-invariant zero temperature limit. Around the deconfinement temperature $T_{c}$, the density $\rho_{t}$ of intersection points with a plane running in the time and one space direction be- 
gins to decrease and retains roughly a third of its zero temperature magnitude at $T=2 T_{c}$. The density $\rho_{t}$ is relevant for the behavior of the Polyakov loop correlator, since the area between two Polyakov loops extends in the time and one space direction. However, since $\rho_{t}$ does not vanish for $T>T_{c}$, deconfinement cannot be simply ascribed to the behavior of $\rho_{t}$, i.e. the partial polarization of the vortices in time direction. Instead, more detailed correlations between the intersection points building up the density $\rho_{t}$ must be present.

By contrast, the behavior of the density $\rho_{s}$ of intersection points with planes running in two spatial directions largely parallels the known behavior of the spatial string tension $\sigma_{s}$ [22], [21]. After a slight dip around $T_{c}$, which may be a finite-size artefact, $\rho_{s}$ begins to rise with temperature along with the spatial string tension $\sigma_{s}$. This is consistent with a simple picture of a random distribution of the intersection points making up the density $\rho_{s}$. In such a picture, the associated (spatial) string tension is proportional to the density [9].

Returning to the question of the deconfinement transition, we measured in more detail the probability that an even or an odd number of intersection points occurs on the area spanned by two Polyakov loops at a large distance from one another (half the lattice universe). This quantity exhibits the deconfinement transition very clearly, which is not surprising, since (up to a trivial shift) it corresponds to the value of the Polyakov loop correlator itself, cf. eq. (3). This is a slightly more physical realization of the usual Polyakov loop order parameter, namely, instead of putting one static color source on the lattice and measuring the associated free energy, we give the color source a static partner at the edge of the universe. Thus, we have not really defined a new order parameter, but the vortex picture provides an alternative language for describing the Polyakov loop correlator: The deconfinement transition occurs when the intersection points of vortices with the area spanned by the Polyakov loops begin to occur predominantly in pairs of finite separation (above $T_{c}$ ), whereas they occur randomly below $T_{c}$. We conjecture this behavior to be connected with a percolation transition in the vortex surfaces: Since the vortex surfaces are closed, intersection points of these surfaces with the entire plane containing two Polyakov loops must always occur in pairs. What determines the behavior of the Polyakov loop correlator is whether the members of a pair are only separated by a distance small compared with the separation of the Polyakov loops, such that most pairs lie either entirely inside or outside the area spanned by the Polyakov loops, or whether the members of a pair can occur arbitrarily far apart, such that the distribution of points is essentially random. In terms of the vortex surfaces, this translates into the question whether typical vortex clusters have a finite extension, or whether they form networks extending over the entire universe, i.e. percolate. This leads to the conjecture that the confining phase is a phase in which the vortices percolate throughout space-time, while in the deconfining phase, they cease to do so. We 
plan to report on a detailed examination of the vortex percolation properties in an upcoming publication.

Acknowledgements. M.E. acknowledges a useful discussion with F.Karsch on the static quark potential at finite temperatures. This work was supported in part by DFG under contract Re 856/1-3.

\section{References}

[1] S. Mandelstam, Phys. Rep. 23C (1976) 245;

G. 't Hooft, Nucl. Phys. B190 (1981) 455.

[2] A. Kronfeld, M. Laursen, G. Schierholz and U.-J. Wiese, Nucl. Phys. B293 (1987) 461.

[3] T. Suzuki and I. Yotsuyanagi, Phys. Rev. D 42 (1990) 4257.

[4] M. I. Polikarpov, Nucl. Phys. Proc. Suppl. 53 (1997) 134.

[5] L. Del Debbio, M. Faber, J. Greensite and Š. Olejník, Phys. Rev. D 55 (1997) 2298.

[6] L. Del Debbio, M. Faber, J. Greensite and Š. Olejník, talk presented at the NATO Advanced Research Workshop on Theoretical Physics: New Developments in Quantum Field Theory, Zakopane, Poland, 14-20 June 1997, hep-lat/9708023.

[7] L. Del Debbio, M. Faber, J. Giedt, J. Greensite and Š. Olejník, Phys. Rev. D 58 (1998) 094501, hep-lat/9801027.

[8] K. Langfeld, H. Reinhardt and O. Tennert, Phys. Lett. B419 (1998) 317.

[9] M. Engelhardt, K. Langfeld, H. Reinhardt and O. Tennert, Phys. Lett. B431 (1998) 141.

[10] J. Fingberg, U. Heller and F. Karsch, Nucl. Phys. B392 (1993) 493.

[11] G. 't Hooft, Nucl. Phys. B138 (1978) 1.

[12] Y. Aharonov, A. Casher and S. Yankielowicz, Nucl. Phys. B146 (1978) 256.

[13] J. M. Cornwall, Nucl. Phys. B157 (1979) 392;

J. M. Cornwall, hep-th/9712248. 
[14] J. Ambjørn and P. Olesen, Nucl. Phys. B170 [FS1] (1980) 265;

P. Olesen, Nucl. Phys. B200 [FS4] (1982) 381.

[15] G. Mack, in "Recent developments in gauge theories", eds. G. 't Hooft et al. (Plenum, New York, 1980);

G. Mack and E. Pietarinen, Nucl. Phys. B205 [FS5] (1982) 141.

[16] E. T. Tomboulis, Phys. Lett. B303 (1993) 103;

T. G. Kovács and E. T. Tomboulis, hep-lat/9711009.

[17] B. Svetitsky, Phys. Rep. 132 (1986) 1. (1982) 423.

[18] O. Kaczmarek, Diploma thesis, Bielefeld 1997.

[19] F. Karsch and C. B. Lang, Phys. Lett. B138 (1984) 176.

[20] C. DeTar, O. Kaczmarek, F. Karsch and E. Laermann, Phys. Rev. D 59 (1999) 031501.

[21] G. S. Bali, J. Fingberg, U. M. Heller, F. Karsch and K. Schilling, Phys. Rev. Lett. 71 (1993) 3059.

[22] T. Appelquist and R. D. Pisarski, Phys. Rev. D 23 (1981) 2305. 